\title{
Control Oriented Reduced Order Modeling of a Flexible Winged Aircraft
}

\author{
Tamás Luspay \\ Systems and Control Lab \\ Institute for Computer Science and Control \\ Kende 13-17, Budapest, Hungary 1111 \\ luspay.tamas@sztaki.mta.hu
}

\author{
Tamás Péni \\ Systems and Control Lab \\ Institute for Computer Science and Control \\ Kende 13-17, Budapest, Hungary 1111 \\ peni.tamas@sztaki.mta.hu
}

\author{
Bálint Vanek \\ Systems and Control Lab \\ Institute for Computer Science and Control \\ Kende 13-17, Budapest, Hungary 1111 \\ vanek.balint@sztaki.mta.hu
}

\begin{abstract}
This paper presents a systematic framework for developing a dynamical model for flexible aircrafts, which is suitable for model-based control design. Traditional modeling tools, such as Finite Element (FEM) and Doublet Lattice Method (DLM) are used for developing a detailed model, which captures the entire behavior of the aircraft. However, the dimension and complexity of the model exceed the currently available computational need of model-based control design. Consequently, a model order reduction framework has been developed and applied for the high fidelity representation. The paper is the first reported results on reducing a 524 dimensional dynamical aircraft model. The main focus is to preserve the information relevant for control design, e.g., the flutter phenomena. From this viewpoint, the final 35 dimensional model shows encouraging results, as shown in the paper.
\end{abstract}

\section{TABle of Contents}

1. INTRODUCTION $\ldots \ldots \ldots \ldots \ldots \ldots \ldots \ldots \ldots \ldots \ldots \ldots \ldots \ldots$.

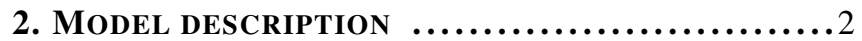

3. MODEL ORDER REDUCTION $\ldots . . . \ldots \ldots \ldots \ldots . . . \ldots 4$

4. NUMERICAL RESULTS $\ldots . . . . \ldots \ldots \ldots . . . \ldots \ldots 6$

5. SUMMARY AND OUTLOOK ........................ 7

ACKNOWLEDGMENTS ...............................8 8

REFERENCES ......................................8

BIOGRAPHY ......................................9

\section{INTRODUCTION}

The future trends in aircraft design are oriented to build more lightweight aircrafts in order to increase fuel efficiency and decrease the operating costs. To achieve these goals the decrease of the structural mass and the use of more flexible components are currently investigated by researchers in the field [1] and [2]. This paper presents the results and status of the ongoing FLEXOP project (EU Horizons No 636307) in terms of the model based control design.

The main challenge related to flexible aircraft is the occurrence of the flutter phenomena during normal operation conditions. This is due to aeroelastic effects, where at certain airspeed the natural frequencies of different dynamical modes become coupled, leading to the loss of stability. Consequently, active control methods are needed to expand the flight envelope above the flutter speed.

978-1-5386-2014-4/18/\$31.00 (C)2018 IEEE

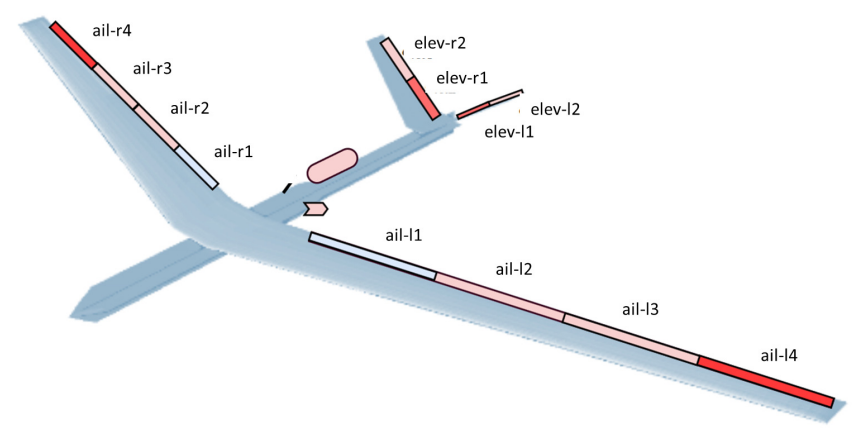

Figure 1. Control surface configuration.

In order to successfully design a controller, which suppresses these adverse aeroelastic effects, a suitable dynamic model is needed [3]. Aeroservoelastic models can be constructed based on a subsystem approach [4] first a linear structural model is generated by finite element method (FEM) method, rigid body dynamics are replaced by non-linear equations of motion and then it is interconnected with a linear unsteady aerodynamic model generated by Double Lattice Method (DLM) [5]. Since the structural damping changes with increasing airflow speed, the model is obtained at different airspeed as a linear structure, hence the dynamics are in a linear-parameter varying (LPV) form. In order to capture the relevant aeroelastic effects an accurate model is needed, which requires the use of a suitably dense structural grid (155 structural grid points) and large number (480) of lag states in the aerodynamic model. This results in a high-dimensional dynamical system with 524 state variables. Unfortunately this is intractable by the currently available analysis and control synthesis algorithms developed for LPV systems [6],[7]. This makes it necessary to develop an appropriate model order reduction method, which finds a lower dimensional representation for the same dynamical behavior.

The paper is organized as follows. After the introduction we turn our attention on the development of the model. Section 2 discusses the modeling process to obtain a dynamical model of the flexible winged aircraft. In the following Section 3 we briefly summarize our recently developed LPV model reduction technique. The interested reader is referred to [8], [9] for more details on the algorithm and to [10] and [11] for application examples. However, the present paper shows the reduction of a more complex model, therefore Section 4 is fully dedicated to the numerical results. 


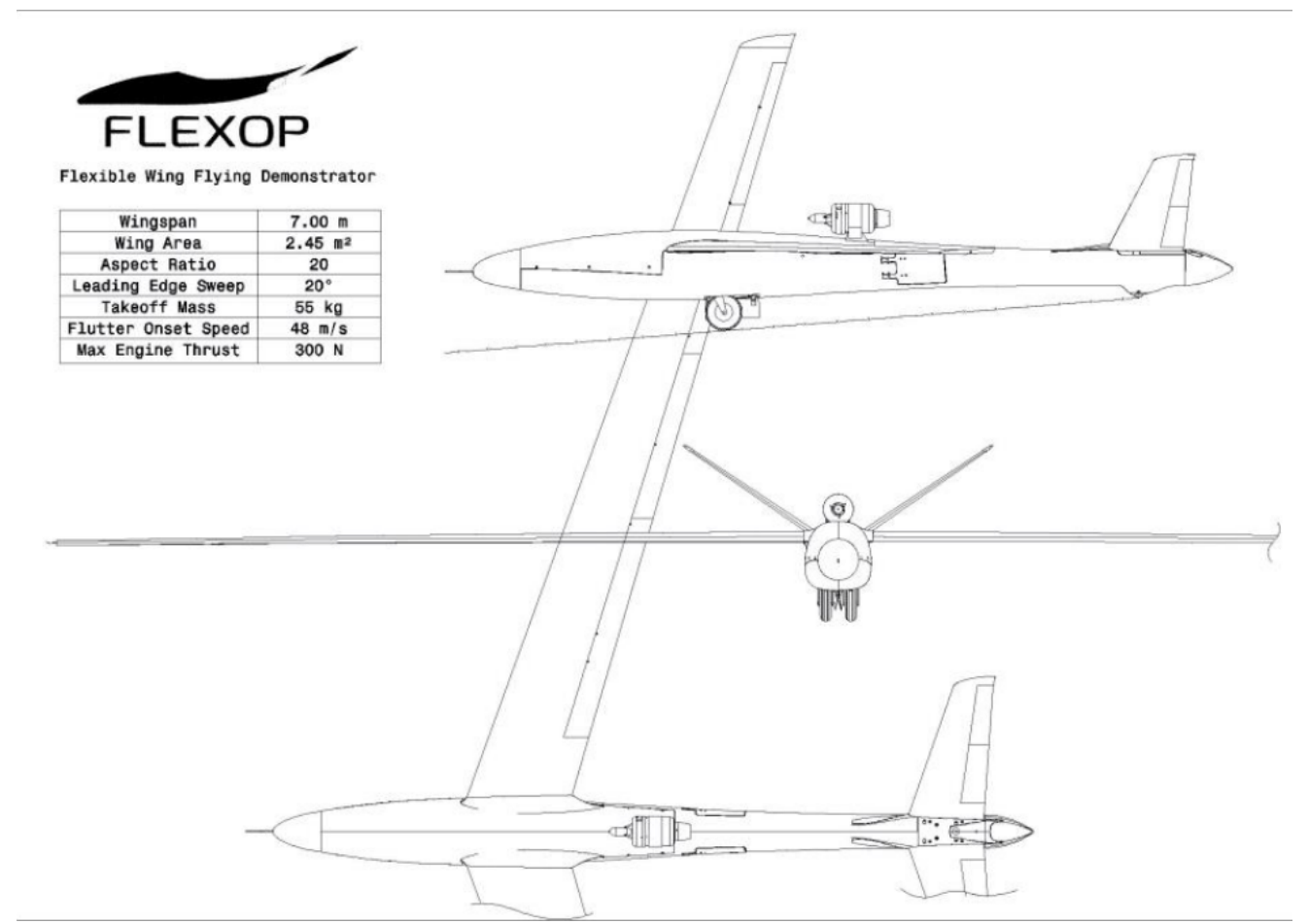

Figure 2. The FLEXOP demonstrator UAV

\section{MODEL DESCRIPTION}

The FLEXOP demonstrator UAV is illustrated in Figure 2. The design features a wing span of 7 meters at an aspect ratio of 20 . The takeoff weight is typically $55 \mathrm{~kg}$ but can be increased by up to $11 \mathrm{~kg}$ of ballast. The aircraft is equipped with a $300 \mathrm{~N}$ jet engine, located on the fuselage back. An air-brake system, deflecting from the sides of the fuselage, enables fast deceleration, fast airspeed control and steep approach angles. The empennage is configured as a V-tail, while each wing half features four control surfaces of which the outermost one is used for flutter suppression (see Fig 1). A custom made actuator moves the surface with sufficient bandwidth. The two innermost control surfaces serve as high lift devices during takeoff and landing. The aircraft has two flutter modes. The first one gets unstable at $48.1 \frac{\mathrm{m}}{\mathrm{s}}$ and $7.95 \mathrm{~Hz}$, when the second bending and symmetric torsion modes are coupled. The second flutter mode follows at $50.5 \frac{\mathrm{m}}{\mathrm{s}}$ and $6.42 \mathrm{~Hz}$ as an antisymmetric first bending form. Divergence occurs at $62.5 \frac{\mathrm{m}}{\mathrm{s}}$.

The aeroservoelastic dynamical model of the aircraft is developed by the German Aerospace Center, DLROberpfaffenhofen, based on a subsystem approach as outlined in the sequel [12][4]. The aerodynamics and the structural dynamics are developed separately and the interconnection forms the aeroservoelastic model (see Fig. 3).

\section{Equations of Motion}

First a finite element model (FEM) is created to form the equations of motion for the aircraft [13]. The FEM model goes under a modal decomposition and Guyan reduction [14],

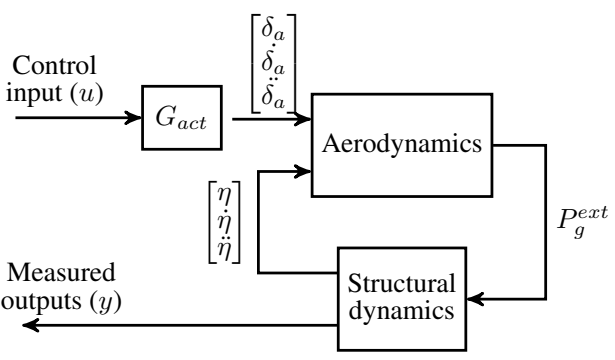

Figure 3. Aeroelastic model

and a linear form is obtained:

$$
\begin{aligned}
& \left\{-\omega^{2}\left[\begin{array}{cc}
M_{b b} & 0 \\
0 & M_{f f}
\end{array}\right]+i \omega\left[\begin{array}{cc}
0 & 0 \\
0 & B_{f f}
\end{array}\right]+\right. \\
& \left.\left[\begin{array}{cc}
0 & 0 \\
0 & K_{f f}
\end{array}\right]\right\}\left[\begin{array}{l}
\eta_{b} \\
\eta_{f}
\end{array}\right]=\left[\begin{array}{l}
\Phi_{g b}^{T} \\
\Phi_{g f}^{T}
\end{array}\right] P_{g}^{e x t}(\omega)
\end{aligned}
$$

Equation (1) is explicitly split into a rigid body and flexible part (denoted by the subscripts $b$ and $f$ ) with modal matrices $\Phi_{g b}$ and $\Phi_{g f}$ respectively. Additionally $M, B$ and $K$ are the modal mass, damping and stiffness matrices respectively and $P_{g}^{e x t}$ is the external excitation in modal coordinates. For the FLEXOP aircraft a 6 degree-of-freedom rigid body was used along with the flexible part consisting of 16 modes.

The linear model in (1) is only valid for small perturbations, therefore the rigid body part is replaced by a non-linear one, describing the movement relative to a mean axes body reference frame. A common element in such applications is the Euler-Bernoulli-beam with added torsional effects. Based on some simplifying assumptions [4], the following form is 
obtained:

$$
\begin{aligned}
& {\left[\begin{array}{c}
m_{b}\left(\dot{V}_{b}+\Omega_{b} \times V_{b}-T_{b E} g_{E}\right) \\
J_{b} \dot{\Omega}_{b}+\Omega_{b} \times J_{b} \Omega_{b}
\end{array}\right]=\Phi_{g b}^{T} P_{g}^{e x t}(t) } \\
& M_{f f} \ddot{\eta}_{f}+B_{f f} \dot{\eta}_{f}+K_{f f} \eta_{f}=\Phi_{g f}^{T} P_{g}^{e x t}(t)
\end{aligned}
$$

where $V_{b}$ and $\Omega_{b}$ are the velocity and angular velocity in the body frame. The mass distribution of the wing is assumed to be replaced by a concentrated mass system based on physical considerations. The 165 structural grid points, including the control surface deflections, are placed forward and after along the concentrated masses. The structural grid points have 6 degrees of freedom.

The external forces $P_{g}^{e x t}$ acting on the structure are dependent on the rigid body and flexible motion, as well as the atmospheric disturbances. The calculation of these unsteady aerodynamic forces is carried out by the Doublet Lattice Method (DLM) [5].

\section{Aerodynamic forces}

The unsteady aerodynamics is modeled with the subsonic Doublet Lattice Method [5], where the model is divided into aerodynamic panels. A short summary of the generalized aerodynamic model for the aerodynamic panels is given based on [15], [16].

The DLM results in the $A I C$ (Aerodynamic Influence Coefficient) matrices that relate the normal-wash vector $\bar{w}$ to the normalized pressure difference vector $\bar{p}$ about the panels as

$$
\bar{p}_{\text {panel }}=\left[A I C_{\text {panel }}(\omega, V)\right] \bar{w}
$$

where $\omega$ is the oscillating frequency and $V$ is the air speed. These two parameters are generally transformed into a single dimensionless parameter, the reduced frequency: $k=\frac{\omega \bar{c}}{2 V}$, where $\bar{c}$ is the reference chord length. In order to relate the modal displacements to the normal-wash vector $\bar{w}$ and to transform the aerodynamic force to modal coordinates the so called generalized aerodynamic matrix (GAM) is defined as (see [15], [16] for more details)

$$
Q_{\text {panel }}(k)=\Phi^{T} T_{a s}^{T} S\left[A I C_{\text {panel }}(k)\right]\left(D_{1}+i k D_{2}\right) T_{a s} \Phi
$$

where $D_{1}$ and $D_{2}$ are the differentiation matrices, $S$ is the integration matrix and $T_{a s}$ is the interpolation matrix that projects the structural grid deformation on to the aerodynamic panels in form of their pitch and heave deformation [4]. The GAM maps the modal deformation $\eta$ to the aerodynamic force distribution in modal coordinates. Note that the GAM matrices are obtained only over a discrete reduced frequency grid. However, time domain aeroelastic simulations require a continuous model. There are several methods to obtain such models [17], among these Roger's rational function approximation (RFA) method [17] was applied for the underlying aircraft model. The resulting aerodynamic model is obtained in the form

$$
\begin{aligned}
Q_{\text {panel }}(k)= & Q_{\text {panel }_{0}}+Q_{\text {panel }_{1}} i k+Q_{\text {panel }_{2}}(i k)^{2}+ \\
& \sum_{l=1}^{n_{p}} Q_{\text {panel }_{l+2}} \frac{i k}{i k+b_{l}}
\end{aligned}
$$

where $Q_{\text {panel }_{0}}, Q_{\text {panel }_{1}}$ and $Q_{\text {panel }_{2}}$ stand for the quasisteady, velocity and acceleration terms of the aerodynamic model. The $Q_{\text {panel }_{l+2}}$ terms take the lag behavior of the aerodynamic model into account. The poles of the lag states are given by $b_{l} . n_{p}$ number of poles are selected for each modal coordinate a priori. In order to appropriately capture the aerodynamic behavior $n_{p}=30$ poles have been selected for the FLEXOP aircraft. This implies that the resulting aerodynamic model is of much higher dimension than the structural model. At the same time, it is also clear that from a input-output point of view, the behavior can be well approximated with smaller dimensions, which will be later revealed by the model reduction algorithm. In a similar fashion, the GAM matrices for the control surface deflection $\delta_{a}$ can be also defined.

\section{Model summary}

The rigid body motion is modeled through the classical 6 degree-of-freedom description, which implies 12 state variables:

$$
x_{\text {rigid }}=\left[\begin{array}{llllllllllll}
u & v & w & p & q & r & \phi & \theta & \psi & x & y & z
\end{array}\right]^{T},
$$

where $u, v, w$ are the body frame velocities, $p, q, r$ are the roll, pitch and yaw rates respectively, while $\phi, \theta, \psi$ are the roll, pitch and heading angles. The structural dynamics of the aircraft contains the first 16 structural modes and their time derivatives, which corresponds to a 32 state model. The aerodynamic model is constructed by selecting $n_{p}=30$ poles for each structural coordinate. Therefore, the aerodynamic model consists of 480 lag states, and the final model has 524 state variables.

As illustrated in Figure 1, the aircraft has 12 control surfaces. Each control surface deflection together with its derivative and second derivative enters the model as control input, representing 36 input channels. Together with the additional thrust and air-brake signals the final model formulation has alltogether 38 inputs.

The output signals of the aircraft can be divided into two groups. We assume that traditional sensors, i.e. accelerometers, rate gyros, pressure sensors and magnetometers provide rigid body related information. More specifically:

$$
y_{\text {rigid }}=\left[\begin{array}{llllllllll}
u & v & w & p & q & r & \phi & \theta & M A C H & \beta
\end{array}\right]^{T}
$$

are available through measurements and can be used for synthesizing rigid body control laws. In addition to these measurements, dedicated flutter sensors will be also installed on the demonstrator aircraft. Accelerometers are placed at different cross sections of the wing on the forward and after structural grid points. Figure 4 shows possible configurations, from which only a few will be used. The optimal sensor selection for flutter suppression is currently in the focus of our research. In the present study we only used the $z$ directional acceleration and $x, y$ directional angular velocities of sensors $L 6$ and $R 6$, located at the tip of the wings. That is:

$$
y_{\text {flutter }}=\left[\begin{array}{llllll}
a_{z}^{L 6} & r_{x}^{L 6} & r_{y}^{L 6} & a_{z}^{R 6} & r_{x}^{R 6} & r_{y}^{R 6}
\end{array}\right]^{T} .
$$

Consequently, the dimension of the measured output vector is 16.

Finally, the developed non-linear model has been trimmed and linearized at $N=26$ different air speed values between 45 and $70 \frac{\mathrm{m}}{\mathrm{sec}}$, giving birth to a Linear Parameter Varying system model [18], [19]:

$$
G(\rho): \begin{aligned}
& \dot{x}(t)=A(\rho(t)) x(t)+B(\rho(t)) u(t) \\
& y(t)=C(\rho(t)) x(t)+D(\rho(t)) u(t)
\end{aligned}
$$




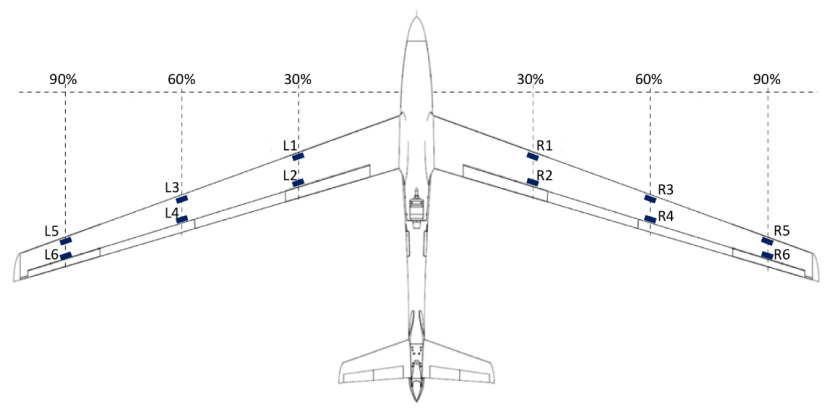

Figure 4. Flutter sensor configuration.

with a grid-based representation:

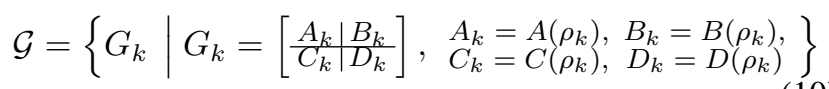

Here, the scheduling parameter $\rho$ is the airspeed defined in the interval $\Gamma:=\left[\begin{array}{ll}45 & 70\end{array}\right] \mathrm{m} / \mathrm{s} . \quad A_{k} \in \mathbb{R}^{524 \times 524}, B_{k} \in$ $\mathbb{R}^{524 \times 38}, C_{k} \in \mathbb{R}^{16 \times 524}$ and $D_{k} \in \mathbb{R}^{16 \times 38}$ are the system matrices obtained from the linearization around trim point $\rho_{k}$ $k=1, \ldots, N$.

The complexity and dimension of the developed description represent the main obstacle for exploiting the virtues of the described modeling chain and consequently using it for controller design [3]. A model order reduction methodology is developed and applied to offer a remedy.

\section{MOdel ORder Reduction}

The model order reduction algorithm, recently developed for Linear Parameter Varying (LPV) systems can be subdivided into several methodological blocks. The detailed algorithm can be found in [20], [9], hereunder we only provide a brief overview for the readers.

We consider LPV dynamics given as a set of Linear Time Invariant (LTI) systems obtained over a suitably dense parameter grid (as in (10)). Our aim is to find a lower order approximant of system, which can be used for designing a model based controller. Accordingly, the dynamical properties and input-output behavior of the full order model has to be preserved as much as possible [21].

At the first stage of the algorithm, the grid-based LPV model (10) is transformed into a parameter-varying modal form. The motivation originates from the modal decomposition of LTI systems, where the model is reformulated as an interconnected series of independent dynamics.

\section{Modal form of LTI systems}

For LTI systems in modal form the structure of the $A$ matrix is block-diagonal:

$$
A=\left[\begin{array}{cccc}
A_{1} & 0 & 0 & \ldots \\
0 & A_{2} & 0 & \\
\vdots & & \ddots & 0 \\
& & 0 & A_{m}
\end{array}\right]
$$

Here each block is composed from the corresponding eigenvalue of the system $^{2}$, i.e. $A_{i}=\lambda_{i}$ if $\lambda_{i} \in \mathbb{R}$ and $A_{i}=$ $\left[\begin{array}{cc}\mathfrak{R e}\left(\lambda_{\mathfrak{i}}\right) & \mathfrak{I m}\left(\lambda_{\mathfrak{i}}\right) \\ -\mathfrak{I m}\left(\lambda_{\mathfrak{i}}\right) & \mathfrak{R e}\left(\lambda_{\mathfrak{i}}\right)\end{array}\right]$ if $\lambda_{i} \in \mathbb{C}$. It is known, that the similarity state transformation, which produces the decoupled form can be constructed from the eigeinvectors of the $A$ matrix as follows:

$$
T_{m}=\left[\begin{array}{lllll}
\nu_{1} & \ldots & \nu_{i} & \ldots & \nu_{m}
\end{array}\right]^{-1},
$$

where $\nu_{i}=\mathfrak{R e}\left(v_{i}\right)$ if $\lambda_{i} \in \mathbb{R}$ and $\nu_{i}=\left[\begin{array}{ll}\mathfrak{R e}\left(v_{i}\right) & \mathfrak{I m}\left(v_{i}\right)\end{array}\right]$ otherwise, i.e. the modal form requires the eigendecomposition of matrix $A$. This feature is not favorable in the model reduction context, due to the bad numerical conditioning of the large scale eigenvalue problem [22]. Therefore, a preliminary conditioning, such as diagonal scaling, has to be carried out for the system (10) before computing the eigenvalue-eigenvector pairs [22].

When extending the idea of modal decomposition for parameter varying systems, one has to face at least two important problems [23]. Firstly, the consistency of the state-space, i.e. the correct ordering of the modal blocks, must be ensured over the entire parameter domain [19]. This requires the tracking of the modes between subsequent grid points. Secondly, the parameter-varying modal transformation should have a smooth parameter dependence (differentiable) in order to facilitate the smooth interpolation of the modal (and reduced) model.

So, our aim is to construct the differentiable $\lambda_{i}(\rho), v_{i}(\rho)$ functions satisfying $\lambda_{i}\left(\rho_{k}\right)=\lambda_{k, i}$ and $v_{i}\left(\rho_{k}\right)=v_{k, i}$, where $\Lambda_{k}=\operatorname{blockdiag}\left(\lambda_{k, 1}, \ldots, \lambda_{k, n_{x}}\right)$ and $V_{k}=\left[v_{k, 1}, \ldots, v_{k, n_{x}}\right]$ define the eigen-decomposition of $A_{k}$ such that

$$
\Lambda_{k}=V_{k}^{-1} A_{k} V_{k}, \quad k=1 \ldots N .
$$

\section{Modal form of LPV systems}

Connecting the dynamical modes over the parameter domain, to ensure state-space consistency, is formulated as a minimum cost perfect matching over a bipartite graph [9].

Eigenvalues at a certain gridpoint $k$ and at the successive one $k+1$ are considered as two sets of vertices in a graph, where each vertex in $k$ has exactly one pair in $k+1$. The problem is then written as finding the correct pairing between the vertices. For this purpose, the following weighted distance metric is introduced to measure the dynamic similarity between two modes:

$$
h_{w}\left(\lambda_{k, i}, \lambda_{k+1, j}\right):=h\left(\lambda_{k, i}, \lambda_{k+1, j}\right) \cdot\left(1-\left|v_{k, i}^{*} v_{k+1, j}\right|\right) .
$$

$h_{w}$ is constituted by $h$, which measures the distance between $\lambda_{k, i}$ and $\lambda_{k, j}$ and by an extra term characterizing the angle between the corresponding eigenvectors $v_{k, i}$ and $v_{k, j}$. The cost of an edge in the graph then describes the dynamical similarity between the two eigenvalues on the edge. Finding the correct pairing is a minimum cost perfect matching problem [24], which can be solved very efficiently by the Hungarian

${ }^{2} \lambda_{i}$ denotes the $i$-th eigenvalue of matrix $A$, i.e:

$$
A v_{i}=\lambda_{i} v_{i}
$$

where $v_{i}$ is the corresponding eigenvector 
Method or Kuhn-Munkres algorithm in polynomial time [25]. Applying the outlined matching algorithm over the entire parameter domain, the consistent ordering of the modal blocks are achieved.

The next step is to shape the eigenvectors to obtain the desired smoothness along the parameter grid. For technical reasons, multiple eigenvalues and their corresponding eigenspaces are grouped and handled together (see [9] for details). Then each of the $d_{i}$ dimensional eigenvector sequences $V_{1, i}, \ldots, V_{N, i}$ for all $i$, we perform a transformation with an invertible matrix $Q_{k, i}$, which changes the eigenvectors, however leaves the eigenspace intact. For smoothness we require $Q_{k, i}$ to transform the respective eigenvectors at consecutive grid points as close as possible. This condition is formulated as a complex, unconstrained Procrustes problem as follows [26]:

$$
\bar{Q}_{k+1, i}:=\arg \min _{Q_{k+1, i}}\left\|V_{k, i}-V_{k+1, i} Q_{k+1, i}\right\|_{F}, \quad \forall k, i
$$

where $k$ goes from 1 to $N-1, i \in\{1, \ldots, n\}, Q_{k+1, i} \in$ $\mathbb{C}^{d_{i} \times d_{i}}$. Solution of (16) can be given analytically, and the appropriately rotated eigenvectors $\bar{V}_{k+1, i}=V_{k+1, i} \bar{Q}_{k+1, i}$ are then consistent with $V_{k, i}$. The obtained sequence $\bar{V}_{1}, \ldots \bar{V}_{N}$ of the shaped eigenvector matrices (with $\bar{V}_{k}=$ $\left.\left[\begin{array}{lll}\bar{V}_{k, 1} & \ldots & \bar{V}_{k, n}\end{array}\right]\right)$ can be smoothly interpolated over the parameter domain. Consequently, the parameter dependent, differentiable transformation $\bar{T}(\rho)$ can be created similarly to the LTI case (13).

Defining a new state vector $\bar{x}$ such that $\bar{T}(\rho) \bar{x}=x$, the original LPV system (9) transforms into

$$
\begin{aligned}
\dot{\bar{x}} & =\left(\bar{T}^{-1}(\rho) A(\rho) \bar{T}(\rho)-\bar{T}^{-1}(\rho) \frac{\partial \bar{T}(\rho)}{\partial \rho} \dot{\rho}\right) \bar{x} \\
& +\bar{T}^{-1}(\rho) B(\rho) u=(\bar{A}(\rho)+\bar{E}(\rho, \dot{\rho})) \bar{x}+\bar{B}(\rho) u \\
y & =C(\rho) \bar{T}(\rho) \bar{x}+D(\rho) u=\bar{C}(\rho) \bar{x}+D(\rho) u .
\end{aligned}
$$

where $\bar{A}(\rho)$ is the block diagonal part of $\bar{T}^{-1}(\rho) A(\rho) \bar{T}(\rho)$ such that $\bar{A}\left(\rho_{k}\right)=\bar{T}^{-1}\left(\rho_{k}\right) A\left(\rho_{k}\right) \bar{T}\left(\rho_{k}\right)$ for all $\rho_{k} \in \Gamma$. $\bar{E}(\rho, \dot{\rho})$ collects the $\dot{\rho}$-dependent terms in (17) and the difference $\bar{T}^{-1}(\rho) A(\rho) \bar{T}(\rho)-\bar{A}(\rho)$ for all $\rho \in \Gamma$. The latter is zero only at the grid points. The term $\bar{E}(\rho, \dot{\rho})$ represents crosscoupling between the modal subsystems, therefore it has to be handled carefully. However, in many applications the coupling effect can be neglected and the transformed system can be considered fully decoupled, similar to the LTI modal form.

The LPV modal form is state consistent and it is smoothly interpolable over the entire parameter domain furthermore it is a very useful representation in the reduction of large-scale systems, as shown next.

\section{Modal Reduction}

The obtained decoupled structure has three important aspects:

1. Unstable or mixed stability modes (e.g., flutter modes) can be decoupled from the system and accordingly preserved in the reduced order representation. Furthermore, most of the model reduction techniques are mainly applicable for stable systems.

2. Modes outside of the frequency rage of interest can be truncated from the model. This is a very important and useful property in a control oriented reduction framework due to the presence of control bandwidth limitations.
3. The dynamical modes can be easily handled and grouped together to form subsystems with similar (reducible) dynamics. This feature will be exploited in the forthcoming section.

\section{Hierarchical clustering}

The algorithm we have developed so far is able to decouple (at least approximately) the LPV system into a set of independent parameter-varying modal subsystems. Next, the idea is to group the modal blocks with similar dynamical properties into clusters, so that the corresponding larger dimensional subsystems can be efficiently reduced (for more details we refer again to [9]). We propose a hierarchical agglomerative clustering (HAC) framework, as follows. The clustering is based on the eigenvalue trajectories of the LPV system, which have been constructed by the algorithm discussed above. What we need is to compare two eigenvalue trajectories and characterize the similarity in terms of the dynamical response they represent. For this purpose, the following distance metric is introduced between two eigenvalue trajectories $\tau_{i}$ and $\tau_{j}$ :

$H\left(\tau_{i}, \tau_{j}\right)=\min \left(\max _{k} h\left(\lambda_{k, i}, \lambda_{k, j}\right), \max _{k} h\left(\lambda_{k, i}, \lambda_{k, j}^{*}\right)\right)$,

where $h(\cdot, \cdot)$ is the distance function used in (15). Note that the introduced metric (18) ensures merging of complex pairs into one cluster, hence the parameter-varying modes $\left(\tau_{i}, \tau_{i}^{*}\right)$ pairs - take place at the lowest level of our clustering framework [27]. Next, for the comparison of two clusters the complete link clustering is applied: the similarity of two clusters is determined by the similarity of their most dissimilar members [28]. Consequently, in the HAC framework, at each algorithmic step those two clusters are merged together for which the complete link value is the smallest. The merging is repeated until all the objects have been grouped into a single cluster.

The result of the HAC is generally visualized by a dendrogram, which is a tree diagram illustrating how the data objects are merged into larger clusters until the one single cluster is reached. The final cluster structure is obtained by cutting the dendrogram at a user-defined level of similarity. The careful choice of this threshold is important, because it determines the number and size of the clusters generated. In the model reduction framework, this threshold is mainly determined by the available computation capabilities, i.e. the chosen model reduction methodology must be solvable for the largest cluster. In our algorithm, the balanced reduction has been chosen to reduce the dimension of the clusters.

\section{Balanced reduction}

Balanced reduction is a fundamental approach for the model reduction of linear (time invariant and varying, as well as parameter-dependent) systems [21], [29]. The key concept is the balanced realization which reveals the controllability and observability properties of the system. In balanced realization uncontrollable and unobservable states can be identified and deleted easily, without affecting the input-output behavior of the entire system.

Let us assume that after clustering the dynamical modes, $M$ separate LPV systems have been obtained, each of which can be given in the following general form:

$$
\begin{aligned}
& \dot{x}^{(\ell)}=\tilde{A}^{(\ell)}(\tilde{\rho}) \tilde{x}^{(\ell)}+B^{(\ell)}(\tilde{\rho}) u \\
& y^{(\ell)}=\tilde{C}^{(\ell)}(\tilde{\rho}) x^{(\ell)}+D^{(\ell)}(\tilde{\rho}) u,
\end{aligned}
$$




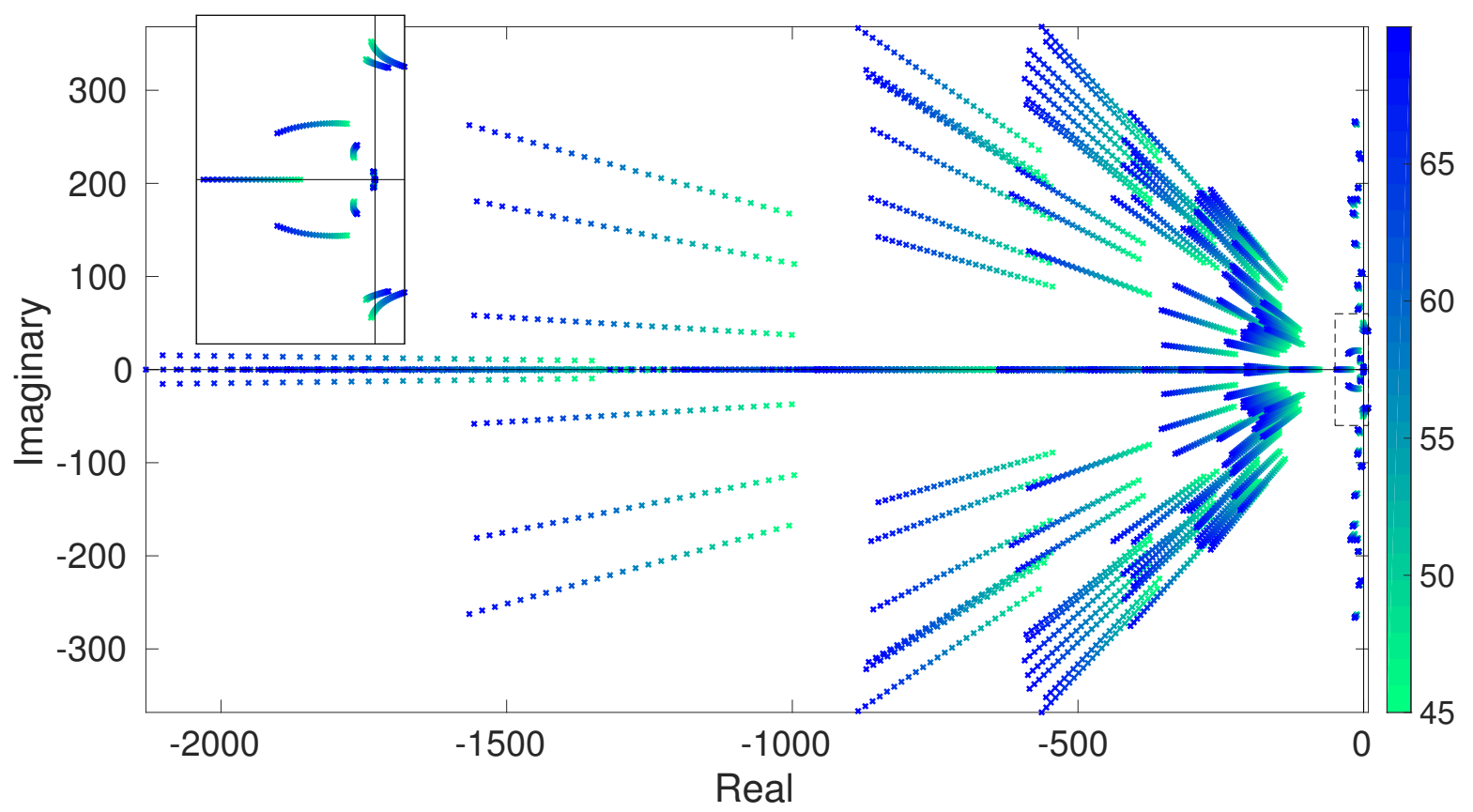

Figure 5. Pole migration of the 524 dimensional system.

where $\tilde{A}^{(\ell)}(\tilde{\rho})=\tilde{A}^{(\ell)}(\rho)+\tilde{E}_{1}(\rho, \dot{\rho})$ and $\tilde{\rho}=[\rho, \dot{\rho}]$. Then, the similarity transformation $\hat{T}(\tilde{\rho})$, which transforms (19) into balanced form can be constructed from the observability $X_{o}^{(\ell)}(\tilde{\rho})$ and controllability $X_{c}^{(\ell)}(\tilde{\rho})$ Gramians [29]. The computation of the Gramians is carried out by the solution of the Lyapunov equality for LTI systems [21] and Lyapunov inequality for LPV system [29].

If the LPV system is given in a state-space form and the structure of the Gramians is a-priori fixed, then $X_{o}^{(\ell)}(\tilde{\rho})$ and $X_{c}^{(\ell)}(\tilde{\rho})$ can be obtained as a result of the following optimization problem [29]:

$$
\begin{array}{r}
\min _{X_{o, i}^{(\ell)}, X_{c, i}^{(\ell)}, i=1 \ldots n_{b}} \sum_{k} \operatorname{trace} X_{o}^{(\ell)}\left(\tilde{\rho}_{k}\right) X_{c}^{(\ell)}\left(\tilde{\rho}_{k}\right) \\
\dot{X}_{o}^{(\ell)}\left(\tilde{\rho}_{k}, \tilde{\nu}_{s}\right)+A^{(\ell)}\left(\tilde{\rho}_{k}\right)^{T} X_{o}^{(\ell)}\left(\tilde{\rho}_{k}\right)+ \\
X_{o}^{(\ell)}\left(\tilde{\rho}_{k}\right) A^{(\ell)}\left(\tilde{\rho}_{k}\right)+C^{(\ell)}\left(\tilde{\rho}_{k}\right)^{T} C^{(\ell)}\left(\tilde{\rho}_{k}\right) \prec 0 \\
-\dot{X}_{c}^{(\ell)}\left(\tilde{\rho}_{k}, \tilde{\nu}_{s}\right)+A^{(\ell)}\left(\tilde{\rho}_{k}\right) X_{c}^{(\ell)}\left(\tilde{\rho}_{k}\right)+ \\
X_{c}^{(\ell)}\left(\tilde{\rho}_{k}\right) A^{(\ell)}\left(\tilde{\rho}_{k}\right)^{T}+B^{(\ell)}\left(\tilde{\rho}_{k}\right) B^{(\ell)}\left(\tilde{\rho}_{k}\right)^{T} \prec 0 \\
X_{o}^{(\ell)} \tilde{\rho}_{k} \succ 0, X_{c}^{(\ell)}\left(\tilde{\rho}_{k}\right) \succ 0, \forall \tilde{\rho}_{k} \in \tilde{\Gamma} \text { and } \forall \tilde{\nu}_{s} \in \tilde{\Omega}
\end{array}
$$

where $\tilde{\Gamma}$ and $\tilde{\Omega}$ are suitably dense grids over $\Gamma \times \Omega$ and $\Omega \times \Phi$, respectively.

This is a nonconvex optimization problem, but if either $X_{o}^{(\ell)}\left(\tilde{\rho}_{k}\right)$ or $X_{c}^{(\ell)}\left(\tilde{\rho}_{k}\right)$ is fixed, then the cost function is linear in the remaining variables, hence the problem reduces to a linear optimization problem with Linear Matrix Inequality (LMI) constraints. As suggested in [29] by alternately fixing $X_{o}^{(\ell)}\left(\tilde{\rho}_{k}\right)$ and $X_{c}^{(\ell)}\left(\tilde{\rho}_{k}\right)$ a numerically tractable iterative algorithm is obtained, where an initial $X_{o}^{(\ell)}\left(\tilde{\rho}_{k}\right)$ (or $\left.X_{c}^{(\ell)}\left(\tilde{\rho}_{k}\right)\right)$ can be calculated from the frozen parameter solutions.
Having determined the observability and controllability Gramians of every subsystem, the balancing $\hat{T}^{(\ell)}(\tilde{\rho})$ transformations and the corresponding parameter dependent, generalized singular value trajectories can be determined. The singular values characterize the controllability and observability properties of the states in the balanced realization. Therefore states with small singular values can be eliminated without affecting the IO behavior. In case the $\hat{T}^{(\ell)}(\tilde{\rho})$ transformations depend on $\rho$ and $\dot{\rho}$, the reduced systems explicitly depend on $\dot{\rho}$ and $\ddot{\rho}$ as well [29]. The details of the related numerical algorithms can be found in [29].

After reducing the subsystems individually, the small dimensional subsystem dynamics are finally joined together with the unstable modes to obtain the low dimensional approximation of (10).

\section{NUMERICAL RESULTS}

As discussed in section 2, the original model consists of 524 states, 38 inputs and 16 outputs given as a set of LTI systems evaluated at 26 airspeed values. The pole migration map of the system is given in Figure 5, where the slow rigid body modes and the flutter modes are zoomed in for better visibility.

There are 4 integrators in the model, closely related to the $x, y, z$ and $\psi$ dynamics of the system. These states have been removed due to numerical reasons. As discussed in 3 our methodology is based on an eigen-decomposition and the corresponding modal transformation. These integrators appear as multiple zero eigenvalues (within machine precision) with an ill-conditoned eigenspace. Therefore keeping them would result in a non-invertible modal transformation (13). If the deleted information is required later then it can be still easily reconstructed from the remaining variables. 

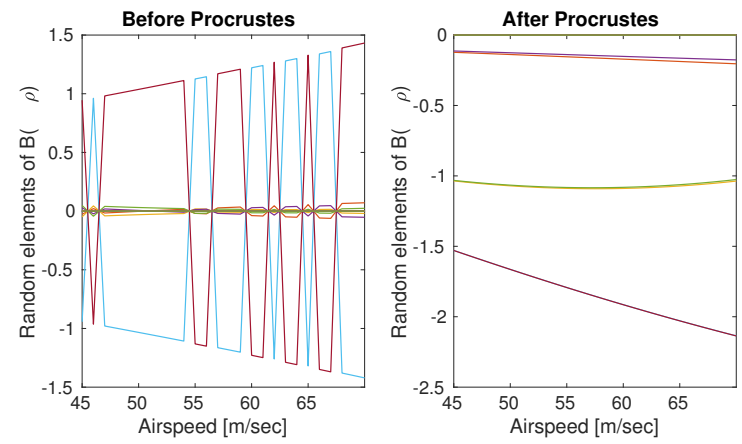

Figure 6. Random entries of $B(\rho)$ in modal forms.

The eigen-decomposition of the 520 dimensional model is then carried out. Multiple eigenvalues, mostly related to the lag state dynamics, are grouped together. The Hungarian algorithm was applied between the grid points to connect the eigenvalue trajectories. Then the Procrustes smoothing was used for the grouped eigenspaces. In order to illustrate the effect of the proposed smoothing, Fig. 6 shows the parameter variation of random entries in $B(\rho)$. It can be clearly depicted that a non-smooth modal transformation results in a non-interpolable LPV system in contrast with the proposed version.

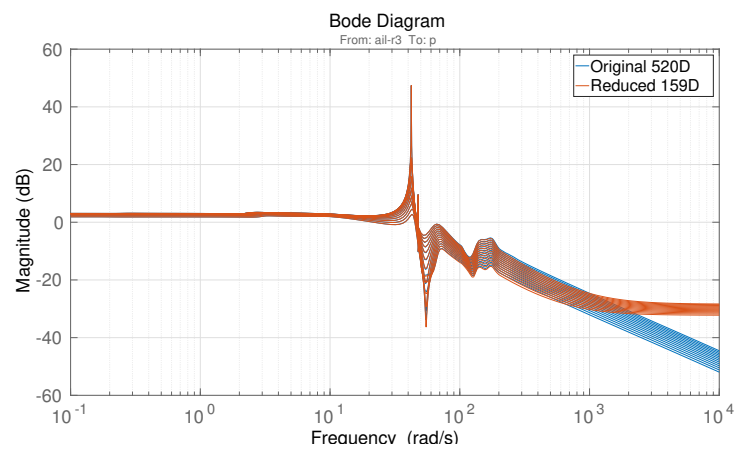

Figure 7. Comparison of frequency response of the original and modal truncated models at different grid points.

Having obtained the smooth LPV modal form we were in the position to remove and store unstable or mixed stability modes. In the underlying system 3 modes have mixed stability properties (flutter and spiral mode), represented by 5 states. These states have been removed and the remaining 515 state then reduced with modal truncation. For the suppression of the flutter phenomena a special, high bandwidth control actuator has been chosen in the demonstrator aircraft. Accordingly a $200 \frac{\mathrm{rad}}{\mathrm{sec}}$ bandwidth has been set for the modal truncation: faster modes have been removed. This step reduced the system to 159 states due to the large number of very fast modes (observe the high frequency modes in Figure 5). The effect of the modal truncation is best illustrated in Figure 7 , where the frequency response from the third right aileron to the roll rate is given. It can be clearly seen that the proposed modal truncation left the lower frequency range intact and preserved the dynamical behavior.

Next, the remaining 159 state have been clustered using the proposed HAC algorithm, which actually revealed that most of the lag-state dynamics can be grouped into clusters. The obtained dendrogram is given in Figure 8. The threshold for

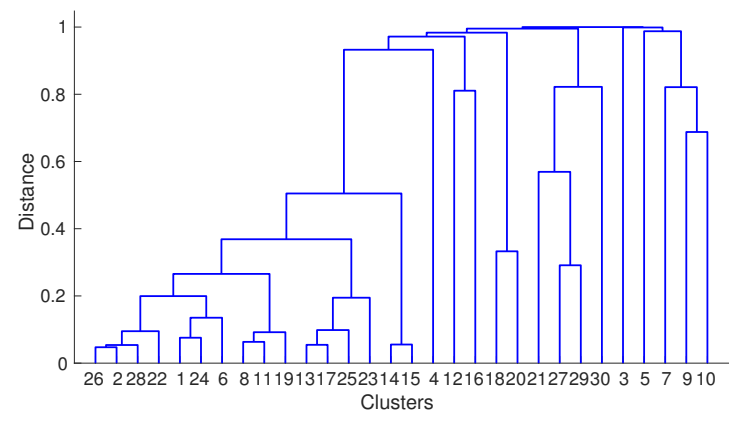

Figure 8. Dendrogram of the clustering.

creating the clusters has been chosen such that the largest cluster's dimension does not exceed 50 .

The controllability and observability Gramians of the smaller dimensional subsystems were computed next. Several configurations were tested with different basis for the Gramians (constant, linear and quadratic). By comparing the generalized singular values we found that parameter-varying Gramians are not giving a significantly lower dimensional approximation than constant ones. Furthermore parameter independent solution of the LMI optimization problem (20) is computationally much easier and the reduced model will not depend explicitly on the parameter change. Accordingly, parameter-independent Gramians were chosen and used for reducing the subsystems.

The reduced systems were then merged together in a 30 dimensional stable LPV model, which is then extended by adding back the 5 dimensional mixed stability part. Consequently, a 35 dimensional approximation has been obtained. Comparison of the pole-migration maps is given in Figure 9. It can be immediately seen which components have been preserved and which ones were compressed and reduced into smaller dimension.

Finally, in order to measure the goodness of the reduced order model we adopted the $\nu$-gap metric [30]. This metric is generally used for characterizing closeness in a closed-loop setup. Since our aim is to use the reduced order model for control design, the $\nu$-gap metric is a suitable choice. Figure 10 shows the frequency distribution of the $\nu$-gap distance. Here, at each frequency we have chosen the worst case value over the parameter domain $\Gamma$ computed between the interpolated models. This is a very important feature, which has to be emphasized.

It can be seen that the distance remains reasonable low for the lower frequency domain and only increases above the prescribed frequency bandwidth used during the modal truncation (compare with Figure 7).

Therefore we conclude that the reduced order approximant of the flexible aircraft can serve as a reliable basis for the control design efforts.

\section{Summary AND OUTLOOK}

We have presented a systematic framework for modeling flexible winged aircrafts and for obtaining its lower order approximant. The results showed that the proposed methodology can be a valuable tool for designing model based 

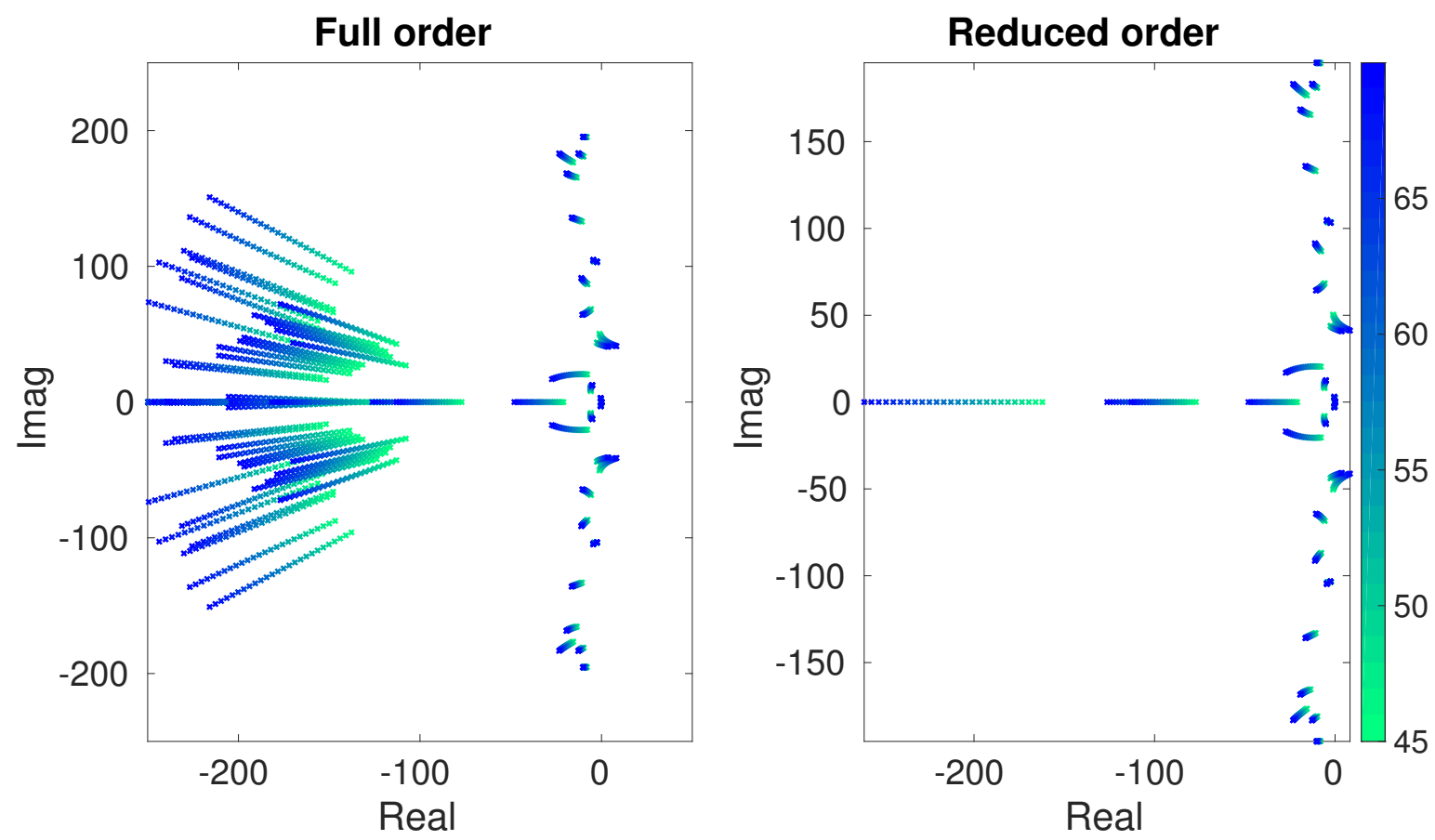

Figure 9. Comparison of pole migration maps.

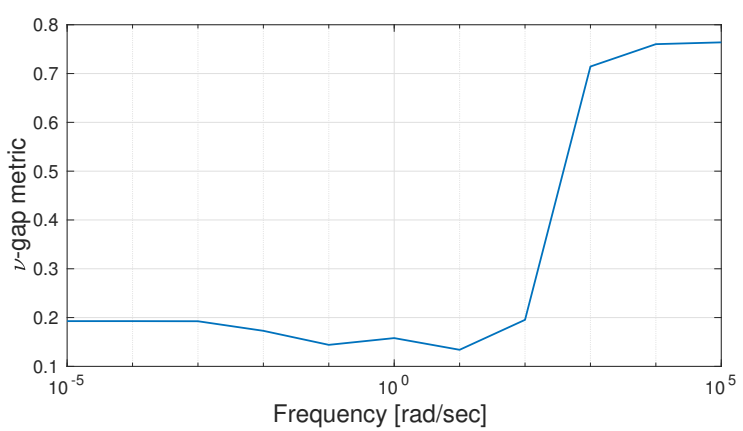

Figure 10. Frequncy distribution of the $\nu$-gap metric between the 520 and 35 order state models.

controllers for aeroelastic systems.

The paper reflects the current status of the FLEXOP project, however we are constantly working on refining and further developing our results. Integration of data from computational fluid dynamics to the model has been performed recently. Meanwhile, gain-scheduled rigid body control laws are designed on the basis of the reduced order model. Flutter suppression controllers are also synthesized and currently being tuned together with the rigid body compensator.

\section{ACKNOWLEDGMENTS}

The research leading to these results is part of the FLEXOP project. This project has received funding from the European Unions Horizon 2020 research and innovation programme under grant agreement No 636307. This paper was supported by the János Bolyai Research Scholarship of the Hungarian Academy of Sciences.

\section{REFERENCES}

[1] FLEXOP, "Flutter Free FLight Envelope eXpansion for ecOnomical Performance improvement (FLEXOP)," Project of the European Union, Project ID: 636307, 2015-2018.

[2] PAAW, "Performance Adaptive Aeroelastic Wing Program," Supported by NASA NRA "Lightweight Adaptive Aeroelastic Wing for Enhanced Perfromace Across the Flight Envelope", 2014-2019.

[3] J. Theis, H. Pfifer, and P. Seiler, "Robust Control Design for Active Flutter Suppression," in AIAA Atmospheric Flight Mechanics Conference, 2016.

[4] T. M. Kier and G. H. N. Looye, "Unifying manoeuvre and gust loads analysis models," in International Forum on Aeroelasticity and Structural Dynamics (IFASD), 2009, pp. 1-20.

[5] E. Albano and W. Rodden, "A doublet-lattice method for calculating lift distributions on oscillating surfaces in subsonic flows," Journal of Aircraft, vol. 7, no. 2, pp. 279-285, 1969.

[6] G. Balas, A. Hjartarson, A. Packard, and P. Seiler, LPVTools: A Toolbox for Modeling, Analysis, and Synthesis of Parameter Varying Control Systems, Musyn, Inc, 2015. [Online]. Available: http://www.aem.umn.edu/ SSeilerControl/software.shtml

[7] M. ApS, The MOSEK optimization toolbox for MATLAB manual, Version 7.1 (Revision 28) ed., 2015. [Online]. Available: http://docs.mosek.com/7.1/toolbox/index.html

[8] I. Gőzse, T. Luspay, T. Péni, Z. Szabó, and B. Vanek, "Model Order Reduction of LPV Systems Based on Parameter Varying Modal Decomposition," in IEEE Conference on Decision and Control, 2016.

[9] T. Luspay, T. Péni, I. Gőzse, Z. Szabó, and B. Vanek, "Model reduction for lpv systems based on approximate 
modal decomposition," International Journal of Numerical Methods in Engineering, vol. Available Online, no. 00, pp. 1-29, 2017.

[10] G. Lipták, T. Luspay, T. Péni, B. Takarics, and B. Vanek, "LPV model reduction methods for aeroelastic structures," in Proceedings of the IFAC World Congress 2017, 2017.

[11] B. Patartics, T. Luspay, T. Péni, B. Takarics, B. Vanek, and T. Kier, "Parameter varying flutter suppression control for the BAH jet transport wing," in Proceedings of the IFAC World Congress 2017, 2017.

[12] T. Kier, G. Looye, M. Scharpenberg, and M. Reijerkerk, "Process, methods and tools for flexible aircraft flight dynamics model integration," in International Forum on Aeroelasticity and Structural Dynamics (IFASD), 2007.

[13] W. P. Rodden, "A method for deriving structural influence coefficients from ground vibration tests." AIAA Journal, vol. 5, no. 5, pp. 991-1000, May 1967.

[14] R. J. Guyan, "Reduction of stiffness and mass matrices," Journal of Aircraft, vol. 2, no. 3, p. 380, 1965.

[15] W. P. Rodden and E. Johnson, "MSC/NASTRAN Aeroelastic Analysis: User's Guide, Version 68," 1994.

[16] A. Kotikalpudi, H. Pfifer, and G. J. Balas, "Unsteady Aerodynamics Modeling for a Flexible Unmanned Air Vehicle," in AIAA Atmospheric Flight Mechanics Conference, 2015.

[17] K. L. Roger, "Airplane Math Modeling Methods for Active Control Design. Structural Aspects of Active Controls," in Structural Aspects of Active Controls, AGARD-CP-228, Aug. 1977, pp. 4-11.

[18] A. Marcos and G. Balas, "Development of linearparameter-varying models for aircraft," Journal of Guidance, Control and Dynamics, vol. 27, no. 2, pp. 218-228, 2004.

[19] C. Poussot-Vassal and C. Roos, "Generation of a reduced-order LPV/LFT model from a set of large-scale MIMO LTI flexible aircraft models," Control Engineering Practice, vol. 20, pp. 919-930, 2012.

[20] I. Gôzse, T. Luspay, T. Péni, Z. Szabó, and B. Vanek, "Model order reduction of LPV systems based on parameter varying modal decomposition," in Conference on Decision and Control (CDC), 2016, pp. 7459-7464.

[21] A. Antoulas, Approximation of Large-Scale Dynamical Systems, ser. Advances in Design and Control. SIAM, 2005.

[22] Y. Saad, Numerical Methods for Large Eigenvalue Problems: Revised Edition, ser. Classics in Applied Mathematics. SIAM, 2011, no. 66.

[23] F. Adegas, I. Sonderby, M. H. Hansen, and J. Stoustrup, "Reduced-order LPV model of flexible wind turbines from high fidelity aeroelastic codes," in IEEE Conference on Control Applications, 2013, pp. 424-429.

[24] R. Burkard, M. Dell'Amico, and S. Martello, Assignment Problems. SIAM, 2009.

[25] L. Lovasz and M. D. Plummer, Matching Theory, ser. Annals of Discrete Mathematics. North-Holland Mathematics Studies, 1986, vol. 29.

[26] D. Amsallem and C. Farhat, "An online method for interpolating linear parametric reduced-order models," SIAM Journal on Scientific Computing, vol. 33, no. 5, pp. 2169-2198, 2011.
[27] T. Hastie, R. Tibshirani, and J. Friedman, The Elements of Statistical Learning, ser. Data Mining, Inference, and Prediction. Springer, 2009.

[28] C. D. Manning and P. R. abd H. Schütze, Introduction to Information Retrieval. Cambridge University Press, 2008.

[29] G. D. Wood, "Control of Parameter-Dependent Mechanical Systems," Ph.D. dissertation, University of Cambridge, 1995.

[30] G. Vinnicombe, "Measuring robustness of feedback systems," Ph.D. dissertation, Department of Engineering, University of Cambridge, 1993.

\section{BIOGRAPHY}

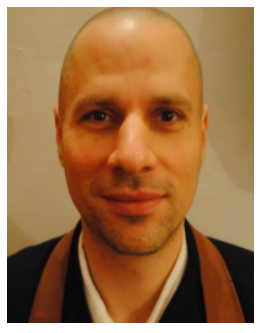

Tamás Luspay received his M.Sc. degree in transportation engineering from the Budapest University of Technology and Economics, in 2006. He holds a Ph.D. in control engineering, received in 2011. He is currently a research fellow at the Systems and Control Lab of the Institute for Computer Science and Control. His main research interest is reduction of large scale systems.

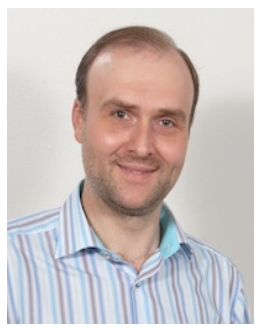

Tamás Péni received his MSc. degree in Electrical Engineering in 1999 and a Ph.D. in Control Engineering from the Budapest University of Technology and Economics in 2009. He is currently a senior research fellow at the Systems and Control Lab of the Institute for Computer Science and Control. His interest include robust control and fault detection of LPV systems.

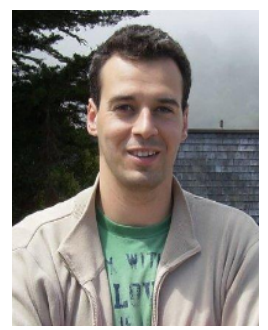

Bálint Vanek received his MSc. degree in transportation engineering in 2003 from the Budapest University of Technology and Economics and a Ph.D. in Control Engineering in 2009 from University of Minnesota. He is currently a senior research fellow at the Systems and Control Lab of the Institute for Computer Science and Control and the leader of the Aerospace Guidance, Navigation, and Control Group. His interest include safety aspects of both manned and unmanned aerial vehicles. 